\title{
Understanding Weight Change while Incarcerated: Qualitative Groundwork for a Collaborative Health Intervention
}

\author{
Nell Baldwin ${ }^{1}$, Amber Cardoos ${ }^{1}$ and Jennifer G. Clarke ${ }^{2 *}$ \\ ${ }^{1}$ Medical Student is affiliated with Warren Alpert Medical School of Brown University, 222 Richmond Street, \\ Providence, RI 02903 \\ ${ }^{2}$ Associate Professor of Medicine and OB/GYN is affiliated with Brown University, Center for Primary Care and \\ Prevention; Memorial Hospital of Rhode Island; Rhode Island Department of Corrections; Alpert Medical School of \\ Brown University; and Center for Prisoner Health and Human Rights. 111 Brewster Street, CPCP bldg. - 2nd Floor, \\ Pawtucket, RI 02860
}

Received: : December 24, 2016; Accepted: January 25, 2017; Published: February 07, 2017

*Corresponding author: Jennifer G. Clarke, Center for Primary Care and Prevention at Memorial Hospital of Rhode Island, 111 Brewster Street, Pawtucket, RI 02860, Tel: 401-729-3400; Fax: 401-729-2494; E-mail:Jennifer_Clarke@brown.edu

\begin{abstract}
In the context of the ever-increasing incarcerated population in the U.S. and our "obesity epidemic," recent work has investigated whether incarcerated individuals are at higher risk for obesity and related diseases. We build on this scholarship by interviewing incarcerated men and women, as well as correctional facility staff to parse the barriers and facilitators to maintaining a healthy weight on the inside. This qualitative analysis forms the groundwork for a collaborative health intervention to address weight gain and weight maintenance in a U.S. correctional facility.
\end{abstract}

Keywords: Qualitative Research; Incarceration; Prison; Obesity; Obesity Prevention; Weight Gain

\section{Introduction}

Addressing the United States' epidemic of obesity is a high priority for preventive and therapeutic interventions [1,2]. Recent work demonstrates both incarcerated men and women are at higher risk for obesity and obesity related diseases [3-8]. This risk may be especially disproportionate for incarcerated juveniles [9] and for incarcerated women [6,10-13]. Some of this work has documented incarceration as an independent risk factor for unhealthy weight status as well as chronic disease burden. Other recent work, for example Bailey et al. [14] study, demonstrate a more complex relationship between incarceration and health status, including obesity. Their work, which contradicts the body of literature cited above, shows an inverse association between incarceration and adult weight gain for men, especially African American men and may point to a protective effect of incarceration for vulnerable populations [14]. This highlights the need for ongoing research and attention to the health effects of incarceration.
Addressing obesity behind bars has increasingly attracted attention from public health researchers and correctional administrators, while still remaining an often elusive goal. Certainly, obesity prevention in any current context has proven challenging [15-18]. In some ways, addressing obesity within the correctional setting offers opportunities, for example, the benefit of the "captive audience" who may not otherwise access healthcare [19]. Finally, the burgeoning costs of managing chronic conditions associated with obesity in the incarcerated setting further engage otherwise unlikely partners for public health interventions [20,21]. For example, the expense of diabetes-related health care has been estimated at $\$ 25,675$ per inmate annually [22].

A health intervention to address obesity within the incarcerated setting could focus on individual behaviorthrough education, one-on-one or group counseling or other tailored programming - or could focus on facility level change such as impacting the foodscape within prison, or other systemic restructuring. Interpersonal counseling, which has been shown to be an effective means to dietary change [23-25], plays a limited role in correctional settings due its high cost. Targeted health education interventions, tailored to the needs of their specific target population, are a promising strategy for addressing obesity in incarcerated populations [26-33]. However, few of these tailored interventions have been developed for low income, ethnically diverse audiences and those adapted for use in correctional settings have not yet been documented to effectively deliver on outcomes [32-34]. One possible reason for the lack of effectiveness of these tailored intervention is a lack of understanding of the specific barriers and facilitators to healthy weight maintenance while incarcerated. Indeed, new participatory based research has offered more successful interventions [35]. 
Facility level interventions remain limited by cost [36] and a sometimes poor understanding of complex systemic barriers and facilitators to healthy weight. "Commissary" typically offers calorie-dense, nutritionally lacking snacks or meal replacements described by Firth et al. describe as "analogous to limited service corner stores" and serves as an example of the complex systemslevel challenges facing interventions [37]. Beyond the financial disincentives in reducing commissary options, there are ethical, financial and security concerns, all of which likely differ between facilities. Despite these many challenges, some successful facility level interventions have been piloted. In Oregon, Firth et al found that reducing the calories served in a women's facility from 3,000 to 2,200 per day while offering increased nutritional education (as part of the Healthy Food Access Project) significantly improved diabetic individuals' glycemic control and did not increase the calories project participants purchased via the commissary [38].

Understanding barriers and facilitators facing incarcerated men and women is of paramount importance when designing tailored programs and when working towards changing the food and exercise environments available in prison [13,35,39-42]. Qualitative research of this kind remains rare, especially research which seeks to find overlap between incarcerated populations and the correctional staff involved in the care of these populations $[40,43]$ While Elwood et al. [35] participatory research, in which incarcerated women designed, led and evaluated a six-week pilot fitness program which resulted in improved body measures and self-reported health benefits [35], offers a visionary model of future directions for this work, this model will not be feasible in many correctional systems. Existing systems level research about prison food and nutrition has traditionally been focused on meeting nutritional standards while limiting costs and complying with security needs $[44,45,38]$.

The purpose of this study is to build the foundation for a collaborative health intervention to address obesity inside the unified correctional facility in Rhode Island. The objectives of this report are (a) to describe the self-reported barriers and facilitators facing male and female inmates in regards to healthy weight maintenance while incarcerated, (b) to interview key staff members at this facility to understand staff perspectives on inmate health and identify logistical concerns and decision makers, (c) to compare solutions and potential interventions proposed by incarcerated individuals with those proposed by staff members, (d) to propose a pilot health intervention informed by the analysis of these qualitative interviews and (e) to discuss programming needs unique to this correctional facility throughout.

\section{Methods}

Study site: The Rhode Island Department of Corrections (RI DOC) is a unified correctional system that serves as a combined prison and jail and holds all of the state's pretrial and sentenced inmates. Smoking is banned at all correctional facilities in the state. We interviewed men from a medium security facility and women from the unified women's facility.

Participants: Research assistants screened men and women in the waiting area associated with the medical offices of their respective buildings. A mix of random and convenience sampling was used-with more of the male participants recruited from in or around the medical offices where the interviews took place. Research assistants called individuals to a private area to explain the study, to assess their eligibility for study participation, and to obtain informed consent if they were eligible and willing to participate. Individuals were told that study participation was completely voluntary and there were no incentives for participation. Institutional review board approval was obtained from Memorial Hospital of Rhode Island prior to initiating the study. The study ran from May 2014 through August 2014. Men were eligible if they were currently housed in the Medium Security facility, had been incarcerated for at least 12 months, were aged 18 years or older, able to complete the interview in English, and able and willing to provide informed consent. Women had similar eligibility criteria, with the exception of any housing restrictions.

Study Protocol: Study participants were interviewed individually, using a topic guide concerned with experiences of weight change in prison, focused on eliciting barriers, facilitators, and solutions. Key staff members were identified through existing contacts of the researchers. Interviews with these key informants followed a topic guide concerned with eliciting the individual's role at this facility, the trends they saw in terms of inmates' weight, knowledge of existing programs, ideas for potential interventions to address the issues they identified. Recruitment continued until saturation was reached with little or no new data or ideas obtained in interviews.

Analysis: All Interviews were audiotaped and transcribed. Two interviewers conducted preliminary analyses on an ongoing basis as each interview transcript was completed. After collection of the interviews, these researchers together identified emerging themes and salient topics of the material and developed an initial codebook. A researcher who was not involved in data collection then conducted in-depth analysis using the immersion/ crystallization method, which involved repeatedly reading the transcripts to identify key themes. The qualitative data analysis software (NVivo) was used to code and organize the data using thematic analysis. Data were subsequently analyzed thematically using the following steps: "becoming familiar with the data;" generating initial codes; applying, editing and consolidating codes; organizing and reviewing themes to generate findings $[40,46]$. Alternative interpretations were discussed before deciding how to report the findings.

\section{Results}

We interviewed 11 women and 17 men who had been incarcerated in Rhode Island for longer than one year. We interviewed four key staff members at the same unified correctional facility who were key decision makers with regards to inmate health.

\section{Perceptions of Weight Gain}

Many male participants reported at least some period of weight gain while incarcerated. Fewer of the female participants 
endorsed periods of weight gain themselves, but suggested that the health of many women in the facility was poor. Staff also reported that unhealthy weight gain occurs among inmates. Male and female participants denied intentional weight gain for any reason, including safety concerns. However, male participants and staff suggested that men may intentionally attempt to gain muscle mass while incarcerated. Female participants and staff observed that women who enter the facility malnourished (often from drug use) may intentionally gain weight upon arrival to reach a healthier weight.

\section{Barriers to and Facilitators of Weight Maintenance}

Motivation: Overall, both men and women suggested that lack of motivation was a major barrier to being active. Participants attributed the lack of motivation to a variety of sources, including the food "slowing them down," lack of knowledge about the health benefits of exercise, and mental health concerns. Healthconscious participants reported that returning home to family was a primary motivator to eating nutritious foods and exercising. While male participants mentioned seeing others getting fit as positive motivation, women mentioned seeing others gain weight as negative motivation to weight maintenance. Several women mentioned exercising with others and exercising for stress relief as motivation to keep active.

Exercise: Many men indicated that lack of mobility due to medical concerns limited their ability to take advantage of the yard or exercise equipment. Examples of medical concerns related to physical health included previous gunshot wounds, low blood sugar, and arthritic joints. In particular, the men indicated that these medical conditions restricted their ability to engage in aerobic exercise. In contrast, nearly all female participants mentioned lack of variety in exercise options as a barrier to exercise. In particular, women noted concerns about limited space and options for exercising. While many women suggested that exercise videos like Zumba are popular, they reported that there is limited space for groups to use the videos. Several women and one staff member noted that inmates may refrain from using the space available due to embarrassment, as the recreation room is located in an open area where correctional officers or other individuals can openly observe.

Several participants, both male and female, suggested that employment within the facility provides opportunities for physical activity. For example, one woman described how she lost weight, despite little time for recreation: "I work in the laundry, sweating like crazy. It's great."

Nutrition: Every participant, both male and female, discussed nutrition as a barrier to weight maintenance while incarcerated. These discussions involved Food Services, which provides three daily meals to the entire population of incarcerated individuals, as well as the commissary, where inmates can purchase items from a store order form on a weekly basis.

Many participants noted that when unappealing food was served in the cafeteria, consumption of commissary food increased. Several described the cafeteria food as "disgusting," and many others described their dislike for the food. For example, one male participant described his experience with the cafeteria food:

"The diet can just make you sick if you're just eating every meal. The first two weeks, I was waiting for money to hit my account and it was like I ate every meal. And for those first two weeks my stomach was messed up. I was just tired all the time. It's not like - the food is not horrible tasting but it just slows you down. You feel slow and after every meal you just want to lay down."

Nearly every participant mentioned that there was too much bread served with meals. Men commented that the overall portions of meals were too small, and many men supplemented their meals with store orders. In contrast, no female participants commented that portions were too small, and many suggested that portions were too large. Nearly every female participant as well as several male participants expressed a desire for more vegetables and salad. One female participant described the changes she would like to see in the cafeteria diet:

"We need double the vegetables. Double the vegetables, half the meat portions - or the fatty portions, whatever they are. And get rid of the white bread. It's as easy as that. It's not really complex. Add some more whole grains - something, some whole grains. Some added fiber - we all struggle with constipation because of the foods. I drink tons and tons of water a day and stay active and I still struggle with constipation just because it's not enough bulk fiber."

Throughout the interviews, male and female participants claimed that the commissary had a negative impact on the health of incarcerated individuals. One female participant summarized the impact of the commissary: "I think [the store order] sabotages every single effort that the nutritionist puts in to diets." Many of the individuals attributed weight gain to the consumption of snack foods purchased through store order, especially in the evening. The most commonly mentioned items purchased from the store order included Ramen noodles, honey buns, meat (including both jerky and "meat logs") and peanut butter. Many participants related this snacking to a form of emotional fulfillment. For example, one male inmate described the relationship of inmates to the commissary: "The ones that can go each week to commissary - they do abuse. We abuse the commissary." The commissary is seen as posing challenges even to those who are physically active:

"Like I be seeing some guys that are going to the gym and you don't see any work on it because they're coming in and eating a bowl with two soups, rice, chili, meat, chips, crackers, cheese. How many calories is that right there? That's like 1000 calorie bowl right there. You just burnt off what, 300, 400 maybe in the gym? If you got enough of a workout in."

Many women described the relationship between the commissary and emotional eating: "The unhealthful foods that come through there are huge and people that are depressed are going to eat more. I mean, ultimately, I think we need things that build people up. We need things that build self-esteem." 
Finally, while some participants mentioned that healthy options do exist on the commissary, one male participant summed up the financial struggle to access healthy food while incarcerated:

"It's hard here because eating healthy in here is expensive. And I mean I got to save my money for other things - I'm saving my money for my daughter, so... And even if you try to be healthy with store orders you're gonna spend 50-60 dollars a week. If you think about it, all that money is junk - stuff that I wouldn't even spend on the outside. Just to get the right amount of protein, spending 10-12 dollars worth of peanuts. They only come in dollar packages, so it's like it's crazy."

Institutional meals and commissary purchases were clearly identified as a barrier to weight maintenance by men, women, and staff. On the other hand, several inmates and staff members compared the diet at these facilities favorably to those in other states.

Food Access: Participants had varied understandings regarding access to and availability of specialized diets. Participants generally recognized that they could meet with the nutritionist to receive a heart healthy diet. However, both men and women expressed doubt at the utility of special diets for health maintenance while the commissary remained an option for less nutritious options.

Access to food was also limited by timing of meals and funds to purchase commissary food. Meals were served around 7:00am, 11:30am, and 4:00pm, and incarcerated individuals were not permitted to bring food outside of the cafeteria for security purposes. Many participants reported eating commissary purchases in the evening. Several participants noted that healthy commissary options tended to be more expensive, while more affordable options (for example, ramen soups) tended to be high in calories, fat, and sodium. For those without resources to make commissary purchases, there was no option for food in the evening. One male participant described the intersection of economics and meals:

"It's very difficult being on population because the last meal, too is at 4:00. And then you don't get nothing else to eat up until 7:00 the next day. So if you have no money you're gonna be hungry and you lose weight."

Finally, access to food could also be determined by employment within the facility. Both female and male participants noted that kitchen workers had increased access to food, though experiences varied on whether this allowed for healthier diets or increased access to high-calorie options.

Mental Health: Nearly every male and female participant associated depression, boredom, and stress with weight gain while incarcerated. Several participants described their time awaiting sentencing as a stressful period associated with weight gain. One male participant described the role of food among inmates who are recently incarcerated: "There's so many emotions going on when you come to jail and food-what do they say-it's like putting a Band-Aid." Another woman described food as a replacement for other addictive substances during a time of boredom: "They're inside here, there's nothing really to do and they don't want to work out, so ... they take something they can use. They can't drink, they can't smoke a cigarette, so they eat food and they blow up." Medications, especially for the treatment of mental illness and diabetes, were also commonly reported as a cause of weight gain.

\section{Solutions and proposals}

Female inmate solutions were largely focused on motivation to exercise, especially by increasing opportunities for recreation and variety in options. Several women noted that these interventions should target whole body health (rather than focus on weight), and should be designed with an emphasis on "fun" and working together towards a goal. More than half of the female participants described changes to the food served in the facility as opportunities for intervention, especially by limiting white bread and starches and increasing consumption of fruit, vegetables, and salad. A few women suggested that good-time incentives would be useful in constructing a weight-loss program at the ACI.

The intervention most frequently suggested by male participants included changes to the food served in the cafeteria, especially to reduce the amount of white bread and other simple carbohydrates served. Healthier options on the commissary list and in the visiting room vending machines were also popular suggestions. Other male participants proposed various programs that would motivate inmates to lose weight or become more active. Some examples of programs suggested included competitive activities (e.g. races) with prizes like store order gift certificates, peer motivation groups with a focus on goal-setting and feedback, shutting down store orders and setting strict diets, and providing consistent annual checks of blood pressure and weight. One participant specifically mentioned that he had found helpful the MOVE! Weight Management Program at the VA, which provides clinician-led group sessions and telephone lifestyle coaching. Despite the frequently mentioned association between mental illness and weight gain, only a few participants suggested interventions based on psychological wellness.

Staff members all supported reducing calories in meals served at the women's facility, especially by limiting servings of white bread. Staff also suggested nutrition education, both targeting short-term strategies while incarcerated and long-term strategies relevant upon release. However, nearly no women mentioned nutrition courses, as they had two regularly-occurring nutrition classes already standard in their programming. Staff and many female participants agreed that an aerobic fitness class would be a popular and feasible intervention for promoting activity.

\section{Discussion}

Several key findings emerged from interviews of inmates and key staff stakeholders in inmate health at these facilities. One theme that emerged was the complementary relationship between inmate and staff perspectives.

First, both inmates and staff agreed that weight gain 
among inmates in the facilities is an issue of concern. Although quantitative evidence of weight gain during incarceration has been documented, to our knowledge this publication is the first to show subjective evidence of and attitudes towards weight change in correctional facilities. Evidence of mutual attention to and concern for an ongoing issue lays the foundation for positive collaboration between inmates, staff, and administration.

Secondly, each inmate and staff member desired change and proposed solutions for interventions that they believed were feasible and targeted to the specific issues at their facility. Moreover, several similar proposals emerged among inmates and staff. For example, all four staff interviewees supported a menu that was specific to the women's facilities, beginning with a reduction in calories. At the time of writing, females and males at this facility were regularly served the same meals with the same caloric content. Many female participants voiced similar suggestions for change, specifically via reduction in calories from simple carbohydrates and larger portions of vegetables. Reassessment of caloric values on menus in a women's correctional facility was successful in Oregon without increasing commissary purchases, further corroborating the usefulness of a nutritional and systemic intervention for weight loss [47]. Efforts to enact similar changes are underway at this women's facility as of the time of this writing.

Information on the diets of incarcerated individuals in the United States is limited. Only two studies to date have published data on a nutritional analysis of foods provided to inmates $[48,49]$. These studies found that menus contained higher levels of cholesterol, sodium, and sugar, and lower levels of fiber, magnesium, potassium, vitamin $\mathrm{D}$, and vitamin $\mathrm{E}$ compared to Dietary Reference Intake recommendations. Additionally, caloric provisions fell below male needs based on standard reference males (92\%) and above female needs based on standard reference females (121\%). However, both analyses were conducted in Southern states whose food costs per inmate per day are significantly lower than those in the facility investigated in this study. Nutritional analysis of the meals served in other, diverse facilities including this facility is warranted to lay the groundwork for intervention development.

At RI DOC, specialized diets are available for inmates with allergic, medical, religious, or other dietary restrictions. In addition, a menu based on the DASH diet is available to those who place a request with the facility's nutritionist. According to staff reports, approximately $5 \%$ of incarcerated individuals at these correctional facilities are on a special diet plan. Despite high rates of obesity, only a small portion of incarcerated individuals consume a diet proven effective for weight reduction, such as the DASH diet [50]. Because serving special meals can be logistically challenging for Food Services, finding ways to incorporate a diet that promotes healthy weight, blood pressure, and cholesterol into the general population menu may be an avenue for future work.

Additionally, male and female participants and staff all agreed that increasing opportunities for exercise would facilitate weight maintenance. Women specifically mentioned increasing the variety in types of exercise available as well as improving the space to exercise, especially by increasing privacy. While both men and women exercise publicly (in front of correctional officers and other inmates), embarrassment was a barrier mentioned by female participants and not by male participants. At the study site, women reported less space and fewer options in exercise equipment compared to men. This may be due to differences in population sizes-in any month, the women's population averages approximately $5 \%$ of the male populationor the building space available to accommodate programming.

Regardless of differences in exercise opportunities available, both male and female participants emphasized the importance of increasing motivation to exercise. Specifically, women proposed a supportive, group-centered program, like an aerobic exercise class. In contrast, men proposed more incentive-based or feedback-based programs, instead of group activities. A program targeting motivation to improve healthy habits around eating and exercising could be tailored to address the needs at either the women's or men's facility. Men and women both mentioned their jobs as their main source of physical activity. The literature supports prison labor as an effective, although ethically challenging, weight management tool [51].Encouragement of physical activity in the workplace may thus be a feasible and desirable opportunity for intervention.

This work examining the facilitators and barriers to weight loss, as well as the solutions proposed by men, women, and staff, has provided the foundation for the development of a weight management intervention. Our group is currently piloting a 6-week intervention in the women's correctional facility that provides low-cost resources for building healthy habits in exercise and nutrition. The intervention will consist of weekly group meetings facilitated by research staff to educate, provide support, and reflect on progress. If successful, this low-cost, lowresource intervention could be tailored to other correctional settings. Other work includes reducing the number of calories to recommended levels for the female population within this facility and altering the content of the cafeteria meals.

This study is limited by the non-random sample of volunteers. For male participants, recruitment was largely based on individuals who came to the clinic and self-selected to participate. Thus the male voices included in this work may represent a "sicker" population. Random sampling for men and women with stays longer than one year was also performed. By locating the interviews within medical office space, participants' knowledge of and comfort in medical settings may have contributed to biased self-selection on the part of our study participants. The findings were based on participant reports so accuracy cannot be verified, and differences in findings among participants cannot be reconciled. All interviews were conducted in the same correctional system over a 6-month period, and generalizability may be thus limited.

These findings have established a foundation for scientific 
testing of weight loss interventions that will be desirable, feasible, and acceptable within the constraints of a correctional facility.

\section{Acknowledgements}

This project was funded by the Predoctoral Training in Primary Care and Family Medicine Grant from the Health Resources \& Services Administration (HRSA) in the form of a 2014 Summer Assistantship from Alpert Medical School of Brown University, as well as a 2016 Summer Assistantship from Brown University. Dr. Fadya El Rayess provided invaluable guidance throughout all stages of the project. Kira Bromwich assisted with interview transcription and Katalin Spremulli provided vital logistical support and expertise. The RI DOC staff for their ongoing support.

\section{References}

1. The Surgeon General's call to action to prevent and decrease overweight and obesity: Rockville, MD: US Department of Health and Human Services, Public Health Service, Office of the Surgeon General; 2001.

2. Ogden CL, Carroll MD. Prevalence of overweight, obesity, and extreme obesity among adults: United States, trends 1960-1962 through 2007-2008. National Center for Health Statistics. 2010;6:1-6.

3. Binswanger IA, Krueger PM, Steiner JF. Prevalence of chronic medical conditions among jail and prison inmates in the USA compared with the general population. J Epidemiol Community Health. 2009;63(11):9129. doi: $10.1136 /$ jech.2009.090662

4. Houle B. Obesity disparities among disadvantaged men: National adult male inmate prevalence pooled with non-incarcerated estimates, United States, 2002-2004. Soc Sci Med. 2011;72(10):1667-73. doi 10.1016/j.socscimed.2011.03.039.

5. Fogel CI. Hard time: the stressful nature of incarceration for women. Issues Ment Health Nurs. 1993;14(4):367-77.

6. Clarke JG, Waring ME. Overweight, obesity, and weight change among incarcerated women. J Correct Health Care. 2012;18(4):285-92. doi: $10.1177 / 1078345812456010$

7. Brennan VM, Kumanyika SK, Zambrana RE. Obesity Interventions in Underserved Communities: Evidence and Directions: JHU Press; 2014.

8. Schnittker J, John A. Enduring Stigma: The Long-Term Effects of Incarceration on Health. Journal of Health and Social Behavior. 2007;48(2):115-130.

9. Robinson KM, Haupt-Hoffman D, Stewart B, Schneider F, Hamm N, Garrison V. Is obesity a problem in a juvenile correctional facility? Journal of Correctional Health Care. 2006;12(3):175-180.

10. Massie JA. Changes in weight experienced by female inmates in the Federal Bureau of Prisons: DTIC Document; 2000.

11. Gates ML, Bradford RK. The Impact of Incarceration on Obesity: Are Prisoners with Chronic Diseases Becoming Overweight and Obese during Their Confinement? Journal of obesity. 2015;2015.

12. Leigey ME, Johnston ME. The Prevalence of Overweight and Obesity Among Aging Female Inmates. J Correct Health Care. 2015;21(3):27685. doi: $10.1177 / 1078345815588171$

13. Shaw NS, Rutherdale M, Kenny J. Eating more and enjoying it less: US prison diets for women. Women Health. 1985;10(1):39-57.
14. Bailey ZD, Williams DR, Kawachi I, Okechukwu CA. Incarceration and adult weight gain in the National Survey of American Life (NSAL). Prev Med. 2015;81:380-6. doi: 10.1016/j.ypmed.2015.09.015

15. Befort CA, Nollen N, Ellerbeck EF, Sullivan DK, Thomas JL, Ahluwalia JS. Motivational interviewing fails to improve outcomes of a behavioral weight loss program for obese African American women: a pilot randomized trial. J Behav Med. 2008;31(5):367-77. doi: 10.1007/ s10865-008-9161-8

16. Hanson C, Gray G. Lessons learned from developing a women's prison health promotion program. Journal of Health Education. 1997;28(6):374-377.

17. Boon CS, Clydesdale FM. A review of childhood and adolescent obesity interventions. Crit Rev Food Sci Nutr. 2005;45(7-8):511-25.

18. Economos CD, Irish-Hauser S. Community interventions: a brief overview and their applications to the obesity epidemic. J Law Med Ethics. 2007;35(1):131-7.

19. Khavjou OA, Clarke J, Hofeldt RM, Lihs P, Loo RK, Prabhu M, et al. A captive audience: bringing the WISEWOMAN program to South Dakota prisoners. Womens Health Issues. 2007;17(4):193-201.

20. Leddy MA, Schulkin J, Power ML. Consequences of high incarceration rate and high obesity prevalence on the prison system. J Correct Health Care. 2009;15(4):318-27. doi: 10.1177/1078345809340426

21. Glaser JB, Greifinger RB. Correctional health care: a public health opportunity. Ann Intern Med. 1993;118(2):139-45.

22. American Diabetes Association. Economic costs of diabetes in the US in 2012. Diabetes Care. 2013;36(4):1033-46. doi: 10.2337/dc12-2625

23. Contento IR, Randell JS, Basch CE. Review and analysis of evaluation measures used in nutrition education intervention research. J Nutr Educ Behav. 2002;34(1):2-25.

24. Ammerman A, Lindquist C, Hersey J, Jackman AM, Gavin NI, Garces C, et al. Efficacy of Interventions to modify dietary behavior related to cancer risk. Evid Rep Technol Assess. 2000;(25):1-4.

25. Räsänen M, Niinikoski H, Keskinen S, Helenius H, Talvia S, Rönnemaa $\mathrm{T}$, et al. Parental nutrition knowledge and nutrient intake in an atherosclerosis prevention project: the impact of child-targeted nutrition counselling. Appetite. 2003;41(1):69-77.

26.Campbell MK, DeVellis BM, Strecher VJ, Ammerman AS, DeVellis RF, Sandler RS. Improving dietary behavior: the effectiveness of tailored messages in primary care settings. Am J Public Health. 1994;84(5):783-7.

27. Glasgow RE, Toobert DJ, Hampson S. Effects of a brief office-based intervention to facilitate diabetes dietary self-management. Diabetes Care. 1996;19(8):835-842.

28. Scisney-Matlock M, Glazewki L, McClerking C, Kachorek L. Development and evaluation of DASH diet tailored messages for hypertension treatment. Appl Nurs Res. 2006;19(2):78-87.

29. Heimendinger J, O’Neill C, Marcus AC, Wolfe P, Julesburg K, Morra $\mathrm{M}$, et al. Multiple tailored messages are effective in increasing fruit and vegetable consumption among callers to the Cancer Information Service. J Health Commun. 2005;10 Suppl 1:65-82.

30. Kreuter MW, Sugg-Skinner C, Holt CL, Clark EM, Haire-Joshu D, et al. Cultural tailoring for mammography and fruit and vegetable intake among low-income African-American women in urban public health centers. Prev Med. 2005;41(1):53-62.

31. Brug J, Steenhuis I, van Assema P, de Vries H. The impact of a computertailored nutrition intervention. Prev Med. 1996;25(3):236-42. 
32. Campbell MK, Tessaro I, DeVellis B, Benedict S, Kelsey K, Belton L, et al. Effects of a tailored health promotion program for female blue-collar workers: health works for women. Prev Med. 2002;34(3):313-23.

33. Kroeze W, Werkman A, Brug J. A systematic review of randomized trials on the effectiveness of computer-tailored education on physical activity and dietary behaviors. Ann Behav Med. 2006;31(3):205-23.

34. De Bourdeaudhuij I, Stevens V, Vandelanotte C, Brug J. Evaluation of an interactive computer-tailored nutrition intervention in a real-life setting. Ann Behav Med. 2007;33(1):39-48.

35. Elwood Martin R, Adamson S, Korchinski M, Granger-Brown A, R Ramsden V, A Buxton J, et al. Incarcerated women develop a nutrition and fitness program: participatory research. Int J Prison Health. 2013;9(3):142-50. doi: 10.1108/IJPH-03-2013-0015

36. Stein K. Foodservice in correctional facilities. J Acad Nutr Diet. 2000;100(5):508-509. doi: 10.1016/S0002-8223(00)00153-X.

37. Firth CL, Drach L, Maher JE, Peters CS. Lack of Healthier Food Alternatives Can Compromise Inmate Health. Am J Public Health. 2015;105(6):e4-5. doi: 10.2105/AJPH.2015.302666

38. Firth CL, Sazie E, Hedberg K, Drach L, Maher J. Female Inmates with Diabetes: Results from Changes in a Prison Food Environment. Womens Health Issues. 2015;25(6):732-8. doi: 10.1016/j.whi.2015.07.009

39. Gately C, Bowen A, Kennedy A, MacDonald W, Rogers A. Prisoner perspectives on managing long term conditions: a qualitative study. International Journal of Prisoner Health. 2006;2(2):91-99.

40.Smoyer AB, Blankenship KM. Dealing food: Female drug users' narratives about food in a prison place and implications for their health. Int J Drug Policy. 2014;25(3):562-8. doi: 10.1016/j. drugpo.2013.10.013

41. Condon L, Hek G, Harris F, Powell J, Kemple T, Price S. Users' views of prison health services: a qualitative study. Journal of advanced nursing. J Adv Nurs. 2007;58(3):216-26.

42. Smoyer AB. Good and Healthy: Foodways and Construction of Identity in a Women's Prison. The Howard Journal of Criminal Justice. 2014;53(5):525-541.

43. Condon L, Hek G, Harris F. Choosing health in prison: Prisoners' views on making healthy choices in English prisons. Health Education Journal. 2008;67(3):155-166.

44. Eves A, Gesch B. Food provision and the nutritional implications of food choices made by young adult males, in a young offenders' institution. J Hum Nutr Diet. 2003;16(3):167-79.

45. Nikolas H. The Well Women Project: Meeting women's nutrition needs at the Adelaide Women's Prison. Women in Corrections, Adelaide, Australia. 2000

46. Tammam J, Gillam L, Gesch B, Stein J. Availability of junk food should be reduced. BMJ. 2012;345:e7070. doi: 10.1136/bmj.e7070.

47. Braun V, Clarke V. Using thematic analysis in psychology. Qualitative Research in Psychology. 2006;3(2):77-101.

48. Cook EA, Lee YM, White BD, Gropper SS. The Diet of Inmates: An Analysis of a 28-Day Cycle Menu Used in a Large County Jail in the State of Georgia J Correct Health Care. 2015;21(4):390-9. doi: $10.1177 / 1078345815600160$

49. Collins SA, Thompson SH. What are we feeding our inmates? J Correct Health Care. 2012;18(3):210-8. doi: 10.1177/1078345812444875

50.Soltani S, Shirani F, Chitsazi MJ, Salehi-Abargouei A. The effect of dietary approaches to stop hypertension (DASH) diet on weight and body composition in adults: a systematic review and meta-analysis of randomized controlled clinical trials. Obes Rev. 2016;17(5):442-54. doi: $10.1111 /$ obr.12391 